\title{
Analysis of a Stochastic Competitive Model with Distributed Time Delays and Jumps in a Polluted Environment
}

\author{
Taolin Zhang $(\mathbb{D}$, Yuanfu Shao $\mathbb{D}$, and Xiaowan She $\mathbb{D}$ \\ College of Science, Guilin University of Technology, Guilin, Guangxi 541004, China \\ Correspondence should be addressed to Yuanfu Shao; shaoyuanfu@163.com
}

Received 16 December 2020; Revised 25 January 2021; Accepted 30 January 2021; Published 15 February 2021

Academic Editor: Maria Alessandra Ragusa

Copyright ( 2021 Taolin Zhang et al. This is an open access article distributed under the Creative Commons Attribution License, which permits unrestricted use, distribution, and reproduction in any medium, provided the original work is properly cited.

In this paper, a stochastic competitive model with distributed time delays and Lévy jumps is formulated. With or without a polluted environment, the model is denoted by $(M)$ or $\left(M_{0}\right)$, respectively. The existence of positive solution, persistence in mean, and extinction of species for $(M)$ and $\left(M_{0}\right)$ are both studied. The sufficient criteria of stability in distribution for model $(M)$ is obtained. Finally, some numerical simulations are given to illustrate our theoretical results.

\section{Introduction}

The dynamics of the biological system has attracted many researchers and has no interruption in the past few decades. This includes the study of the persistence and extinction, stability in distribution of biological systems, optimal harvesting effects of renewable resources (for example, fish and plants), and so on. These studies have implications for the management of biological resources. The dynamics behaviors from the initial deterministic model to stochastic model have been extensively studied and a lot of nice results have been reported [1-4]. It has been verified that the growth rates of species are inevitably subject to white noise. And whether to consider the white noise is the difference between the stochastic model and the deterministic model. Following the method adopted in [4], we will model a stochastic system with white noise. For the biological system, usually there are three kinds of population relationship, i.e., predator-prey, mutualistic, and competitive scenarios, where the competitive scenario between populations is relatively popular $[5,6]$. The general competitive model between two populations with white noise is as follows:

$$
\left\{\begin{array}{l}
\mathrm{d} y_{1}(t)=y_{1}(t)\left[r_{10}-a_{11} y_{1}(t)-a_{12} y_{2}(t)\right] \mathrm{d} t+\sigma_{1} y_{1}(t) \mathrm{d} B_{1}(t) \\
\mathrm{d} y_{2}(t)=y_{2}(t)\left[r_{20}-a_{21} y_{1}(t)-a_{22} y_{2}(t)\right] \mathrm{d} t+\sigma_{2} y_{2}(t) \mathrm{d} B_{2}(t)
\end{array}\right.
$$

where $y_{i}(t)$ is the size of the $i$-th population at time $t ; r_{i 0}$ represents the growth rate of $i$-th population; $a_{i i}>0$ denotes the intraspecific competitive coefficients of $y_{i} ; a_{12}$ and $a_{21}$ are positive and represent the competitive rates between $y_{1}$ and $y_{2}$, respectively; $B_{i}(t)$ stands for the standard Brownian motion defined on a complete probability space $\left(\Omega, \mathscr{F},\{\mathscr{F}\}_{t \geq 0}, P\right)$ with a filtration $\{\mathscr{F}\}_{t \geq 0}$ satisfying the usual conditions (i.e., it is increasing and right continuous and $\mathscr{F}_{0}$ contains all $P$ null sets); $\sigma_{i}^{2}$ is the intensity of the white noise, $i=1,2$.

However, the world economy is developing more and more rapidly and the economic development will inevitably destroy the ecological environment. With the increasing toxins and pollution into the ecological environment, the quality of human living environment is becoming worse and worse. Therefore, the study on the impact of toxin 
importation and environmental pollution on biological populations has become one of the most popular topics in the world [7-12], which is of vital significance to the development of sustainable economy and the protection of human's only living environment. Based on model (1) and considering the environmental pollution factors, then we derive the following model:

$$
\left\{\begin{array}{l}
\mathrm{d} y_{1}(t)=y_{1}(t)\left[r_{10}-r_{11} C_{1}(t)-a_{11} y_{1}(t)-a_{12} y_{2}(t)\right] \mathrm{d} t+\sigma_{1} y_{1}(t) \mathrm{d} B_{1}(t) \\
\mathrm{d} y_{2}(t)=y_{2}(t)\left[r_{20}-r_{21} C_{2}(t)-a_{21} y_{1}(t)-a_{22} y_{2}(t)\right] \mathrm{d} t+\sigma_{2} y_{2}(t) \mathrm{d} B_{2}(t) \\
\mathrm{d} C_{1}(t)=\left[k_{1} C_{E}(t)-\left(g_{1}+m_{1}\right) C_{1}(t)\right] \mathrm{d} t \\
\mathrm{~d} C_{2}(t)=\left[k_{2} C_{E}(t)-\left(g_{2}+m_{2}\right) C_{2}(t)\right] \mathrm{d} t \\
\mathrm{~d} C_{E}(t)=\left[-h C_{E}(t)+u(t)\right] \mathrm{d} t
\end{array}\right.
$$

where $C_{1}(t), C_{2}(t)$, and $C_{E}(t)$ are the concentrations of the toxicant in the organism of species $y_{1}$ and $y_{2}$ and environment at time $t$, respectively; $r_{11}$ and $r_{21}$ denote the doseresponse of species $y_{1}$ and $y_{2}$ to the organismal toxicant, respectively; $k_{i}$ and $g_{i}$ represent the absorbing and excretion rates of the toxicant from the environment respectively, $-m_{i}$ is depuration rate of the toxicant, $i=1,2 .-h C_{E}(t)$ denotes the loss rate of the toxicant because of volatilization; $u(t)$ represents the exogenous rate of toxicant inputting into the environment and is always assumed to be bounded.

On the other hand, the behavior between predator and prey is often not always continuous. For example, in some cases, young predators cannot engage in predation; that is, young prey cannot be preyed on. These phenomena are called time delays. Similar phenomena include hibernation, pregnancy, and migration. In fact, time delays exist not only in biological systems, but in other domains as well. For example, R. Manivannan has studied a control system with probabilistic time-delay signals [13]. Therefore, time delays are very important to reveal the real world and should be taken into account in our system. Some scholars pointed out that discrete delays and continuous delays do not include each other, but the S-type distributed delays can be done $[14,15]$. Therefore, taking S-type delays into account in above model is interesting. In addition, in nature there are some environmental perturbations such as earthquakes, epidemics, and hurricanes, which differ from white noise because of its sudden and destructive nature, so Lévy jumps are introduced to simulate them in mathematical modeling [16-21]. For example, Liu and Wang [18] studied the persistence and extinction of the two-species model with Lévy jumps. Liu and Bai [21] investigated the stability in distribution of a stochastic model with Lévy noises by Lyapunov functional approach.

Motivated by these, taking the $S$-type distributed time delays and Lévy noises into the above model, we get the following stochastic predator-prey model $(M)$ :

$$
(M)\left\{\begin{array}{l}
\mathrm{d} y_{1}(t)=y_{1}\left(t^{-}\right)\left[r_{10}-r_{11} C_{1}(t)-a_{11} y_{1}(t)-a_{12} \int_{-\tau_{2}}^{0} y_{2}\left(t^{-}+\theta\right) \mathrm{d} \mu_{2}(\theta)\right] \mathrm{d} t+\sigma_{1} y_{1}(t) \mathrm{d} B_{1}(t) \\
+y_{1}\left(t^{-}\right) \int_{\mathbb{Z}} \gamma_{1}(u) \widetilde{\Gamma}(\mathrm{d} t, \mathrm{~d} u) \\
\mathrm{d} y_{2}(t)=y_{2}\left(t^{-}\right)\left[r_{20}-r_{21} C_{2}(t)-a_{21} \int_{-\tau_{1}}^{0} y_{1}\left(t^{-}+\theta\right) \mathrm{d} \mu_{1}(\theta)-a_{22} y_{2}(t)\right] \mathrm{d} t+\sigma_{2} y_{2}(t) \mathrm{d} B_{2}(t) \\
+y_{2}\left(t^{-}\right) \int_{\mathbb{Z}} \gamma_{2}(u) \widetilde{\Gamma}(\mathrm{d} t, \mathrm{~d} u) \\
\mathrm{d} C_{1}(t)=\left[k_{1} C_{E}(t)-\left(g_{1}+m_{1}\right) C_{1}(t)\right] \mathrm{d} t \\
\mathrm{~d} C_{2}(t)=\left[k_{2} C_{E}(t)-\left(g_{2}+m_{2}\right) C_{2}(t)\right] \mathrm{d} t \\
\mathrm{~d} C_{E}(t)=\left[-h C_{E}(t)+u(t)\right] \mathrm{d} t,
\end{array}\right.
$$

with initial data

$$
\begin{aligned}
y_{i}(\theta) & =\xi_{i}(\theta), \quad \theta \in[-\tau, 0], \\
\tau & =\max \left\{\tau_{i}\right\}, \quad i=1,2,
\end{aligned}
$$

where $y_{i}\left(t^{-}\right)$denotes the left limit of $y_{i}(t)$; $\widetilde{\Gamma}(\mathrm{d} t, \mathrm{~d} u)=\Gamma(\mathrm{d} t, \mathrm{~d} u)-\lambda(\mathrm{d} u) \mathrm{d} t$ represents a compensated
Poisson process, where $\Gamma$ is a Poisson counting measure, $\lambda$ is the characteristic measure of $\Gamma$ on a measurable subset $\mathbb{Z}$ in $(0,+\infty)$ with $\lambda(\mathbb{Z})<+\infty, \lambda(\mathrm{d} u)$ is the measure of $\mathbb{Z} ; \gamma_{i}(u)$ is a bounded function with $\gamma_{i}(u)>-1, u \in \mathbb{Z}$; the term $\int_{-\tau_{i}}^{0} y_{i}\left(t^{-}+\theta\right) \mathrm{d} \mu_{i}(\theta)$ denotes the Lebesgue-Stieltjes integral, where $\mu_{i}(\theta)$ denotes a nonnegative variation function 
defined on $[-\tau, 0]$ with $\tau=\max \left\{\tau_{i}\right\}$ satisfying $\int_{-\tau_{i}}^{0} \mathrm{~d} \mu_{i}(\theta)=$ $1, i=1,2$. The biological meanings of other parameters are the same as before. If $r_{11}=r_{21}=0$, the corresponding model is denoted by $\left(M_{0}\right)$, which means that the population is not contaminated.

We aim to study the dynamical behaviors of $(M)$ and $\left(M_{0}\right)$ such as the extinction and persistence in mean for all species and explore the impacts on the dynamics of time delays and Lévy noise.

The article is structured as follows. For preliminaries, we give some notations and important lemmas in Section 2. In
Section 3, we establish the sufficient criteria for the persistence in mean and nonpersistence of $M$ and $M_{0}$ and investigate the stable in distribution of $(M)$. In Section 4, some numerical simulations are presented to verify our main results. Finally, conclusion and discussion are given to end this article in Section 5.

\section{Preliminaries}

For the simplicity, we first make the following notations:

$$
\begin{aligned}
& b_{i}=r_{i 0}-0.5 \sigma_{i}^{2}-\int_{\mathbb{Z}}\left[\gamma_{i}(u)-\ln \left(1+\gamma_{i}(u)\right) \lambda(\mathrm{d} u)\right], \quad i=1,2, \\
& \left.R_{i}(t)=\int_{0}^{t} \int_{\mathbb{Z}} \ln \left(1+\gamma_{i}(u)\right)\right) \widetilde{\Gamma}(\mathrm{d} s, \mathrm{~d} u), \quad i=1,2, \\
& \overline{f(t)}=t^{-1} \int_{0}^{t} f(s) \mathrm{d} s, \\
& f^{*}=\lim _{t \longrightarrow \infty} \sup f(t), \\
& f_{*}=\lim _{t \rightarrow \infty} \inf f(t), \\
& \overline{f(t)}^{*}=\lim _{t \longrightarrow \infty} \sup t^{-1} \int_{0}^{t} f(s) \mathrm{d} s, \\
& \overline{f(t)}_{*}=\lim _{t \rightarrow \infty} \inf t^{-1} \int_{0}^{t} f(s) \mathrm{d} s, \\
& \Delta=a_{11} a_{22}-a_{12} a_{21}, \\
& \Delta_{1}=a_{22} b_{1}-a_{12} b_{2} \text {, } \\
& \Delta_{2}=a_{11} b_{2}-a_{21} b_{1} \text {, } \\
& \Delta_{31}=a_{22}\left(b_{1}-r_{11}{\overline{C_{1}(t)}}^{*}\right)-a_{12}\left(b_{2}-r_{21}{\overline{C_{2}(t)}}_{*}\right) \text {, } \\
& \Delta_{32}=a_{22}\left(b_{1}-r_{11}{\overline{C_{1}(t)}}_{*}\right)-a_{12}\left(b_{2}-r_{21}{\overline{C_{2}(t)}}^{*}\right) \text {, } \\
& \Delta_{41}=-a_{21}\left(b_{1}-r_{11}{\overline{C_{1}(t)}}_{*}\right)+a_{11}\left(b_{2}-r_{21}{\overline{C_{2}(t)}}^{*}\right) \text {, } \\
& \Delta_{42}=-a_{21}\left(b_{1}-r_{11}{\overline{C_{1}(t)}}^{*}\right)+a_{11}\left(b_{2}-r_{21} \overline{C_{2}(t)}\right) \text {. }
\end{aligned}
$$

Our later discussion are based on the following technological hypotheses.

Assumption 1. There exists a positive constant $L$ such that

$$
\int_{\mathbb{Z}}\left[\ln \left(1+\gamma_{i}(u)\right)\right]^{2} \lambda(\mathrm{d} u)<L, \quad i=1,2 .
$$

Assumption 2. Suppose that $\Delta>0$, which means the internal competition is greater than the external competition (see [22]).

The following lemmas are necessary for our later proof.

Lemma 1. Let Assumption 1 hold, then for any given initial data $\left(\xi_{1}(t), \xi_{2}(t)\right) \in C\left([-\tau, 0], R_{+}^{2}\right)$, there exists a unique solution remaining in $R_{+}^{2}$ with probability 1 .
Proof. It is obvious that the coefficients of model $(M)$ are locally Lipschitz. By [5], model $(M)$ has a unique local solution $y(t)=\left(y_{1}(t), y_{2}(t)\right) \in R_{+}^{2}$ a.s. for any initial data $\left(\xi_{1}(t), \xi_{2}(t)\right) \in C\left([-\tau, 0], R_{+}^{2}\right)$ and $t \in\left[0, \tau_{e}\right]$, where $\tau_{e}>0$ is the explosion time. It needs only to verify that $\tau_{e}=\infty$ a.s. Let $m_{0}>0$ be sufficiently large such that $\left(\xi_{1}(0)\right.$, $\left.\xi_{2}(0)\right) \in\left[1 / m_{0}, m_{0}\right]$ for each integer $m \geq m_{0}$. Define the stopping time

$$
\tau_{m}=\inf \left\{t \in\left[0, \tau_{e}\right]: y_{1}(t) \notin\left(\frac{1}{m}, m\right), y_{2}(t) \notin\left(\frac{1}{m}, m\right)\right\} .
$$

Clearly, $\tau_{m}$ is strictly increasing with $m$. Let $\tau_{\infty}=\lim _{m \longrightarrow \infty} \tau_{m}$ a.s., then $\tau_{\infty} \leq \tau_{m}$ a.s. Then, we only need to prove $\tau_{\infty}=\infty$ a.s. If the statement is not true, then there exist $T>0$ and $0<\varepsilon<1$ such that $P\left(\tau_{\infty}<T\right)>\varepsilon$ and an integer $m_{1}>m_{0}$ such that 


$$
P\left(\tau_{m} \leq T\right)>\varepsilon, \quad \text { for any } m>m_{1} .
$$

Define

$$
V(y)=\beta V_{1}\left(y_{1}\right)+V_{2}\left(y_{2}\right)+V_{3}(t),
$$

where

$$
\begin{aligned}
y & =\left(y_{1}, y_{2}\right), \\
V_{1}\left(y_{1}\right) & =y_{1}-1-\ln y_{1}, \\
V_{2}\left(y_{2}\right) & =y_{2}-1-\ln y_{2}, \\
V_{3}(t) & =\frac{\beta}{2 n^{2}} a_{12} \int_{-\tau_{2}}^{0} \int_{t+\theta}^{t} y_{2}^{2}\left(s^{-}\right) \mathrm{d} s \mathrm{~d} \mu_{2}(\theta)+\frac{a_{21}}{2 n^{2}} \int_{-\tau_{1}}^{0} \int_{t+\theta}^{t} y_{1}^{2}\left(s^{-}\right) \mathrm{d} s \mathrm{~d} \mu_{1}(\theta) .
\end{aligned}
$$

Choose a constant $\beta>0$ and integer $n>0$ such that

For model $(M)$, by Ito's formula, we get

$$
\begin{aligned}
& \frac{a_{21}}{2 n^{2}}-\beta a_{11}<0, \\
& \frac{\beta a_{12}}{2 n^{2}}-a_{21}<0 .
\end{aligned}
$$

$$
\begin{aligned}
\mathrm{d} V(y)= & {\left[\beta L V_{1}\left(y_{1}\right)+L V_{2}\left(y_{2}\right)+\frac{\mathrm{d}}{\mathrm{d} t} V_{3}(t)\right] \mathrm{d} t } \\
& +\beta \sigma_{1}\left(y_{1}-1\right) \mathrm{d} B_{1}(t)+\beta \int_{\mathbb{Z}}\left(y_{1} \gamma_{1}(u)-\ln \left(1+\gamma_{1}(u)\right)\right) \widetilde{\Gamma}(\mathrm{d} s, \mathrm{~d} u) \\
& +\sigma_{2}\left(y_{2}-1\right) \mathrm{d} B_{2}(t)+\int_{\mathbb{Z}}\left(y_{2} \gamma_{2}(u)-\ln \left(1+\gamma_{2}(u)\right)\right) \widetilde{\Gamma}(\mathrm{d} s, \mathrm{~d} u),
\end{aligned}
$$

where

$$
\begin{aligned}
L V_{1}\left(y_{1}\right)= & \left(y_{1}-1\right)\left[r_{10}-r_{11} C_{1}(t)-a_{11} y_{1}\left(t^{-}\right)-a_{12} \int_{-\tau_{2}}^{0} y_{2}\left(t^{-}+\theta\right) d \mu_{2}(\theta)\right] \\
& +0.5 \sigma_{1}^{2}+\int_{\mathbb{Z}}\left(\gamma_{1}(u)-\ln \left(1+\gamma_{1}(u)\right)\right) \lambda(\mathrm{d} u), \\
L V_{2}\left(y_{2}\right)= & \left(y_{2}-1\right)\left[r_{20}-r_{21} C_{2}(t)-a_{21} \int_{-\tau_{1}}^{0} y_{1}\left(t^{-}+\theta\right) \mathrm{d} \mu_{1}(\theta)-a_{22} y_{2}\left(t^{-}\right)\right] \\
& +0.5 \sigma_{2}^{2}+\int_{\mathbb{Z}}\left(\gamma_{2}(u)-\ln \left(1+\gamma_{2}(u)\right)\right) \lambda(\mathrm{d} u) .
\end{aligned}
$$

By basic inequality $a^{2}+b^{2} \geq 2 a b$, we have

$$
\begin{aligned}
& a_{12} \int_{-\tau_{2}}^{0} y_{2}\left(t^{-}+\theta\right) \mathrm{d} \mu_{2}(\theta) \leq \frac{1}{2} a_{12}\left(n^{2}+\frac{1}{n^{2}} \int_{-\tau_{2}}^{0} y_{2}^{2}\left(t^{-}+\theta\right) \mathrm{d} \mu_{2}(\theta)\right), \\
& a_{21} \int_{-\tau_{1}}^{0} y_{1}\left(t^{-}+\theta\right) \mathrm{d} \mu_{1}(\theta) \leq \frac{1}{2} a_{21}\left(n^{2}+\frac{1}{n^{2}} \int_{-\tau_{1}}^{0} y_{1}^{2}\left(t^{-}+\theta\right) \mathrm{d} \mu_{1}(\theta)\right) .
\end{aligned}
$$


Substituting (14) and (15) into $L V_{1}\left(y_{1}\right), L V_{2}\left(y_{2}\right)$, then

$$
\begin{aligned}
L V_{1}\left(y_{1}\right) \leq & y_{1}\left(r_{10}-r_{11} C_{1}(t)\right)-a_{11} y_{1}^{2}-r_{10}+r_{11} C_{1}(t)+a_{11} y_{1}+0.5 \sigma_{1}^{2} \\
& +\int_{\mathbb{Z}}\left(\gamma_{1}(u)-\ln \left(1+\gamma_{1}(u)\right)\right) \lambda(\mathrm{d} u)+a_{12} \int_{-\tau_{2}}^{0} y_{2}\left(t^{-}+\theta\right) \mathrm{d} \mu_{2}(\theta) \\
\leq & y_{1}\left(r_{10}-r_{11} C_{1}(t)\right)-a_{11} y_{1}^{2}-r_{10}+r_{11} C_{1}(t)+a_{11} y_{1}+0.5 \sigma_{1}^{2} \\
& +\int_{\mathbb{Z}}\left(\gamma_{1}(u)-\ln \left(1+\gamma_{1}(u)\right)\right) \lambda(\mathrm{d} u)+\frac{n^{2}}{2} a_{12}+\frac{a_{12}}{2 n^{2}} \int_{-\tau_{2}}^{0} y_{2}^{2}\left(t^{-}+\theta\right) \mathrm{d} \mu_{2}(\theta), \\
L V_{2}\left(y_{2}\right) \leq & y_{2}\left(r_{20}-r_{21} C_{2}(t)\right)-a_{22} y_{2}^{2}-r_{20}+r_{21} C_{2}(t)+a_{22} y_{2}+0.5 \sigma_{2}^{2} \\
& +\int_{\mathbb{Z}}\left(\gamma_{2}(u)-\ln \left(1+\gamma_{2}(u)\right)\right) \lambda(\mathrm{d} u)+\frac{n^{2}}{2} a_{21}+\frac{a_{21}}{2 n^{2}} \int_{-\tau_{1}}^{0} y_{1}^{2}\left(t^{-}+\theta\right) \mathrm{d} \mu_{1}(\theta) .
\end{aligned}
$$

Therefore,

$$
\begin{aligned}
& \beta L V_{1}\left(y_{1}\right)+L V_{2}\left(y_{2}\right)+\frac{\mathrm{d}}{\mathrm{d} t} V_{3}(t) \\
& \leq \beta\left[\begin{array}{c}
\left.r_{10} y_{1}-r_{11} C_{1}(t) y_{1}-a_{11} y_{1}^{2}-r_{10}+r_{11} C_{1}(t)+a_{11} y_{1}+0.5 \sigma_{1}^{2}+\frac{n^{2}}{2} a_{12}\right] \\
+\int_{\mathbb{Z}}\left(\gamma_{1}(u)-\ln \left(1+\gamma_{1}(u)\right)\right) \lambda(\mathrm{d} u)+\frac{a_{12}}{2 n^{2}} y_{2}^{2}
\end{array}\right]+r_{20} y_{2}-r_{21} C_{2}(t) y_{2}-a_{22} y_{2}^{2}-r_{20} \\
& \quad+r_{21} C_{2}(t)+a_{22} y_{2}+0.5 \sigma_{2}^{2}+\int_{\mathbb{Z}}\left(\gamma_{2}(u)-\ln \left(1+\gamma_{2}(u)\right)\right) \lambda(\mathrm{d} u)+\frac{n^{2}}{2} a_{21}+\frac{a_{21}}{2 n^{2}} y_{1}^{2}
\end{aligned}
$$

Substituting (17) into $\mathrm{d} V(y)$ and together with (11), there exists a constant $K>0$ such that

$$
\begin{aligned}
& \mathrm{d} V(y)=K \mathrm{~d} t+\beta \sigma_{1}\left(y_{1}-1\right) \mathrm{d} B_{1}(t)+\beta \int_{\mathbb{Z}}\left(y_{1} \gamma_{1}(u)-\ln \left(1+\gamma_{1}(u)\right)\right) \widetilde{\Gamma}(\mathrm{d} s, \mathrm{~d} u) \\
& \quad+\sigma_{2}\left(y_{2}-1\right) \mathrm{d} B_{2}(t)+\int_{\mathbb{Z}}\left(y_{2} \gamma_{2}(u)-\ln \left(1+\gamma_{2}(u)\right)\right) \widetilde{\Gamma}(\mathrm{d} s, \mathrm{~d} u) .
\end{aligned}
$$

By this result and according to the argument in [23], we have

$$
\infty \leq K T+V_{0}\left(y_{1}(0), y_{2}(0)\right) \leq \infty,
$$

which leads to a contradiction, and hence, $\tau_{\infty}=\infty$ a.s. Therefore, $\tau_{e}=\infty$ a.s. The proof is completed.

Proof. We only prove $\lim _{t \longrightarrow+\infty} \sup E\left[y_{1}^{p}(t)\right] \leq K_{1}(p)$. The rest is similar and omitted. Defining $Q_{1}(t)=e^{t} y_{1}^{p}(t)$, by Ito's formula, we have

$$
\begin{aligned}
\mathrm{d}_{1}(t)= & L Q_{1}(t) \mathrm{d} t+p e^{t} y_{1}^{p} \sigma_{1} \mathrm{~d} B_{1}(t) \\
& +\int_{\mathbb{Z}} e^{t} y_{1}^{p}\left(1+\gamma_{1}(u)\right)^{p} \widetilde{\Gamma}(\mathrm{d} s, \mathrm{~d} u)
\end{aligned}
$$
initial data $\left(\xi_{1}(\theta), \xi_{2}(\theta)\right) \in C\left([-\tau, 0], R_{+}^{2}\right)$. Then, for any $p>0$, there exists a constant $K_{i}(p)>0$ such that 
where

$$
\begin{aligned}
L Q_{1}(t)= & e^{t} y_{1}^{p}\left\{1+\frac{p(p-1) \sigma_{1}^{2}}{2}+\int_{\mathbb{Z}}\left(\left(1+\gamma_{1}(u)\right)^{p}-p \gamma_{1}(u)\right) \lambda(\mathrm{d} u)\right. \\
& \left.+p\left[r_{10}-r_{11} C_{1}(t)-a_{11} y_{1}\left(t^{-}\right)-a_{12} \int_{-\tau_{2}}^{0} y_{2}\left(t^{-}+\theta\right) \mathrm{d} \mu_{2}(\theta)\right]\right\} .
\end{aligned}
$$

Let $K_{1}(p)=\max _{y_{1} \geq 0}\left\{y_{1}^{p}\left[1+\left(p(p-1) \sigma_{1}^{2} / 2\right)+\int_{\mathbb{Z}}((1+\right.\right.$ $\left.\left.\left.\left.\gamma_{1}(u)\right)^{p}-p \gamma_{1}(u)\right) \lambda(\mathrm{d} u)+p r_{10}\right]-p a_{11} y_{1}^{p+1}\right\}$, then

$$
L Q_{1}(t) \leq K_{1}(p) e^{t} .
$$

Integrating both sides of (21) from 0 to $t$ and taking expectation leads to

$$
E\left(e^{t} y_{1}^{p}\right)-\xi_{1}^{p}(0) \leq K_{1}(p)\left(e^{t}-1\right) .
$$

Then, $\lim _{t \rightarrow+\infty} \sup E\left[y_{1}^{p}(t)\right] \leq K_{1}(p)$. The proof is completed.

Lemma 3 (see [18]). Let $Y \in C\left(R_{+} \times \Omega, R_{+}\right), Z \in C\left(R_{+} \times \Omega\right.$, $R)$, and $\rho \in C\left(R_{+}, R\right)$ satisfying $\lim _{t \rightarrow \infty}(Z(t) / t)=0$ a.s.

(1) If there exist two constants $T>0$ and $\rho_{0}>0$ such that $\ln Y(t)=\int_{0}^{t} \rho(s) d s-\rho_{0} \int_{0}^{t} Y(s) d s+Z(t)$ a.s. for all $t \geq T$,

then (i) $\lim _{t \rightarrow \infty} Y(t)=0$ and $\lim _{t \rightarrow \infty}$ sup $(\ln Y(t) / t) \leq 0$ a.s. if $\bar{\rho}<0$;

$\lim _{t \rightarrow \infty} \overline{Y(t)}=\rho / \rho_{0}$ and $\lim _{t \rightarrow \infty}(\ln Y(t) / t)=$ 0 a.s. if $\bar{\rho} \geq 0$.

(2) If there exist two constants $T>0$ and $\rho_{0}>0$ such that $\ln Y(t) \leq \int_{0}^{t} \rho(s) d s-\rho_{0} \int_{0}^{t} Y(s) d s+Z(t)$ a.s. for all $t \geq T$

then (i) $\lim _{t \rightarrow \infty} \overline{Y(t)}^{*} \leq \rho / \rho_{0}$ a.s. if $\bar{\rho} \geq 0$;

$\lim _{t \rightarrow \infty} Y(t)=0$, a.s. if $\bar{\rho}<0$.

(3) If there exist two constants $T>0$ and $\rho_{0}>0$ such that for all

$$
\ln Y(t) \geq \int_{0}^{t} \rho(s) \mathrm{d} s-\rho_{0} \int_{0}^{t} Y(s) \mathrm{d} s+Z(t), \quad \text { a.s. } t \geq T .
$$

Then, $\lim _{t \rightarrow \infty} \overline{Y(t)}_{*} \geq \rho / \rho_{0}$ a.s.

Lemma 4. Let $\left(y_{1}(t), y_{2}(t)\right)$ be any positive solution of model $(M)$, then
(1) $\lim _{t \longrightarrow \infty} \sup \left(\ln y_{i}(t) / t\right) \leq 0$ a.s., $i=1,2$;

(2) For any positive constant $\tau, \quad \lim _{t \rightarrow \infty} t^{-1}$ $\int_{t-\tau}^{t} y_{i}(s) d s=0$ a.s., $i=1,2$.

The proof of Lemma 4 is standard and is omitted (see, e.g., [24]).

\section{Main Results}

3.1. Persistence in Mean

Definition 1 (see [25]). The system is said to be persistence in mean if there are positive constants $v_{i}$ and $s_{i}(i=1,2)$ such that

$$
v_{i} \leq{\overline{y_{i}(t)}}_{*} \leq{\overline{y_{i}(t)}}^{*} \leq s_{i}, \quad i=1,2,
$$

holds for any solution $\left(y_{1}(t), y_{2}(t)\right)$ of model $(M)$ with initial data

$$
y(t)=\left\{\left(\xi_{1}(t), \xi_{2}(t)\right):-\tau \leq t \leq 0\right\} \in C\left([-\tau, 0]: R_{+}^{2}\right) .
$$

Theorem 1. Assume $\Delta_{1}>0, \Delta_{2}>0$, then for any initial data $y(t)=\left\{\left(\xi_{1}(t), \xi_{2}(t)\right):-\tau \leq t \leq 0\right\} \in C\left([-\tau, 0]: R_{+}^{2}\right)$, the solution $\left(y_{1}(t), y_{2}(t)\right)$ of model $\left(M_{0}\right)$ has the following properties:

$$
\begin{aligned}
& \lim _{t \longrightarrow \infty} \overline{y_{1}(t)}=\frac{\Delta_{1}}{\Delta}, \\
& \lim _{t \longrightarrow \infty} \overline{y_{2}(t)}=\frac{\Delta_{2}}{\Delta} .
\end{aligned}
$$

Proof. For $i=1,2$, we compute

$$
\begin{aligned}
& \int_{0}^{t} \int_{-\tau_{i}}^{0} y_{i}\left(s^{-}+\theta\right) \mathrm{d} \mu_{i}(\theta) \mathrm{d} s-\int_{-\tau_{i}}^{0} \mathrm{~d} \mu_{i}(\theta) \int_{0}^{t} y_{i}\left(s^{-}\right) \mathrm{d} s \\
& \quad=\int_{-\tau_{i}}^{0} \int_{\theta}^{0} y_{i}\left(s^{-}\right) \mathrm{d} s \mathrm{~d} \mu_{i}(\theta)-\int_{-\tau_{i}}^{0} \int_{t+\theta}^{t} y_{i}\left(s^{-}\right) \mathrm{d} s \mathrm{~d} \mu_{i}(\theta) .
\end{aligned}
$$


For model $\left(M_{0}\right)$, noticing $\int_{-\tau_{i}}^{0} \mathrm{~d} \mu_{i}(\theta)=1(i=1,2)$ and using Ito's formula, we get

$$
\begin{aligned}
\ln y_{1}(t)= & b_{1} t-a_{11} \int_{0}^{t} y_{1}\left(s^{-}\right) \mathrm{d} s+\sigma_{1} B_{1}(t)+\int_{0}^{t} \int_{\mathbb{Z}} \ln \left(1+\gamma_{1}(u)\right) \widetilde{\Gamma}(\mathrm{d} s, \mathrm{~d} u) \\
& \left.+\ln y_{1}(0)-a_{12} \int_{0}^{t} \int_{-\tau_{2}}^{0} y_{2}\left(s^{-}+\theta\right)\right) \mu_{2}(\theta) \mathrm{d} s \\
= & b_{1} t-a_{11} \int_{0}^{t} y_{1}\left(s^{-}\right) \mathrm{d} s-a_{12} \int_{0}^{t} y_{2}\left(s^{-}\right) \mathrm{d} s+\Phi_{1}(t), \\
\ln y_{2}(t)= & b_{2} t-a_{22} \int_{0}^{t} y_{2}\left(s^{-}\right) \mathrm{d} s-a_{21} \int_{0}^{t} y_{1}\left(s^{-}\right) \mathrm{d} s+\Phi_{2}(t),
\end{aligned}
$$

where

$$
\begin{aligned}
\Phi_{1}(t)= & a_{12} \int_{-\tau_{2}}^{0} \int_{t+\theta}^{t} y_{2}\left(s^{-}\right) \mathrm{d} s \mathrm{~d} \mu_{2}(\theta)-a_{12} \int_{-\tau_{2}}^{0} \int_{\theta}^{0} y_{2}\left(s^{-}\right) \mathrm{d} s \mathrm{~d} \mu_{2}(\theta) \\
& +\ln y_{1}(0)+\sigma_{1} B_{1}(t)+R_{1}(t) \\
\Phi_{2}(t)= & a_{21} \int_{-\tau_{1}}^{0} \int_{t+\theta}^{t} y_{1}\left(s^{-}\right) \mathrm{d} s \mathrm{~d} \mu_{1}(\theta)-a_{21} \int_{-\tau_{1}}^{0} \int_{\theta}^{0} y_{1}\left(s^{-}\right) \mathrm{d} s \mathrm{~d} \mu_{1}(\theta) \\
& +\ln y_{2}(0)+\sigma_{2} B_{2}(t)+R_{2}(t) .
\end{aligned}
$$

Since

$$
\begin{gathered}
\int_{-\tau_{2}}^{0} \int_{t+\theta}^{t} y_{2}\left(s^{-}\right) \mathrm{d} s \mathrm{~d} \mu_{2}(\theta) \leq \int_{-\tau_{2}}^{0} d \mu_{2}(\theta) \int_{t-\tau_{2}}^{t} y_{2}\left(s^{-}\right) \mathrm{d} s, \\
\int_{-\tau_{2}}^{0} \int_{\theta}^{0} y_{2}\left(s^{-}\right) \mathrm{d} s \mathrm{~d} \mu_{2}(\theta) \leq \int_{-\tau_{2}}^{0} \mathrm{~d} \mu_{2}(\theta) \int_{-\tau_{2}}^{0} y_{2}\left(s^{-}\right) \mathrm{d} s .
\end{gathered}
$$

By Lemma 4, we get

$$
\begin{aligned}
& \lim _{t \rightarrow \infty} \int_{-\tau_{2}}^{0} \int_{t+\theta}^{t} y_{2}\left(s^{-}\right) \mathrm{d} s \mathrm{~d} \mu_{2}(\theta)=0, \\
& \lim _{t \rightarrow \infty} \int_{-\tau_{2}}^{0} \int_{\theta}^{0} y_{2}\left(s^{-}\right) \mathrm{d} s \mathrm{~d} \mu_{2}(\theta)=0 .
\end{aligned}
$$

By the same way, we can derive that

$$
\begin{aligned}
& \lim _{t \rightarrow \infty} \int_{-\tau_{1}}^{0} \int_{t+\theta}^{t} y_{1}\left(s^{-}\right) \mathrm{d} s \mathrm{~d} \mu_{1}(\theta)=0, \\
& \lim _{t \rightarrow \infty} \int_{-\tau_{1}}^{0} \int_{\theta}^{0} y_{1}\left(s^{-}\right) \mathrm{d} s \mathrm{~d} \mu_{1}(\theta)=0 .
\end{aligned}
$$

Substituting (35) and (36) into (32) and (33), respectively, then

$$
\begin{aligned}
& \lim _{t \longrightarrow \infty} t^{-1} \Phi_{1}(t)=0, \\
& \lim _{t \longrightarrow \infty} t^{-1} \Phi_{2}(t)=0 \text { a.s. }
\end{aligned}
$$

\section{Computing}

$$
\begin{aligned}
a_{22} & \ln y_{1}(t)-a_{12} \ln y_{2}(t) \\
& =\left(a_{22} b_{1}-a_{12} b_{2}\right) t-\left(a_{11} a_{22}-a_{12} a_{21}\right) \int_{0}^{t} y_{1}\left(s^{-}\right) \mathrm{d} s+\Phi_{3}(t) \\
& =\Delta_{1} t-\Delta \int_{0}^{t} y_{1}\left(s^{-}\right) \mathrm{d} s+\Phi_{3}(t),
\end{aligned}
$$

where $\Phi_{3}(t)=a_{22} \Phi_{1}(t)-a_{12} \Phi_{2}(t)$. From (37), we can easily get $\lim _{t \rightarrow \infty} t^{-1} \Phi_{3}(t)=0$ a.s.

By Lemma 4 again, for any $\varepsilon>0$, there is a $T_{0}>0$ such that

$$
\begin{array}{r}
a_{22} \ln y_{1}(t) \leq\left(\Delta_{1}+a_{12} \varepsilon\right) t-\Delta \int_{0}^{t} y_{1}\left(s^{-}\right) \mathrm{d} s+\Phi_{3}(t), \\
\text { for any } t>T_{0} .
\end{array}
$$

According to Lemma 3, then

$$
\lim _{t \rightarrow \infty}{\overline{y_{1}(t)}}^{*} \leq \frac{\Delta_{1}}{\Delta} \text { a.s. }
$$

Thus, for any $\varepsilon>0$ and sufficiently large $t$, there is

$$
a_{21} \overline{y_{1}(t)} \leq a_{21}{\overline{y_{1}(t)}}^{*}+\varepsilon \leq a_{21} \frac{\Delta_{1}}{\Delta}+\varepsilon
$$


Using (41) in (31), we get

$\ln y_{2}(t) \geq\left(b_{2}-a_{21} \frac{\Delta_{1}}{\Delta}-\varepsilon\right) t-a_{22} \int_{0}^{t} y_{2}\left(s^{-}\right) \mathrm{d} s+\Phi_{2}(t)$

$$
=\left(\frac{a_{22} \Delta_{2}}{\Delta}-\varepsilon\right) t-a_{22} \int_{0}^{t} y_{2}\left(s^{-}\right) \mathrm{d} s+\Phi_{2}(t),
$$

Lemma 3 implies that

$$
\lim _{t \rightarrow \infty}{\overline{y_{2}(t)}}_{*} \geq \frac{\Delta_{2}}{\Delta} \text { a.s. }
$$

Similarly, we have

$$
\begin{aligned}
a_{11} & \ln y_{2}(t)-a_{21} \ln y_{1}(t) \\
& =\left(a_{11} b_{2}-a_{21} b_{1}\right) t-\left(a_{11} a_{22}-a_{12} a_{21}\right) \int_{0}^{t} y_{2}\left(s^{-}\right) \mathrm{d} s+\Phi_{4}(t) \\
& =\Delta_{2} t-\Delta \int_{0}^{t} y_{2}\left(s^{-}\right) \mathrm{d} s+\Phi_{4}(t),
\end{aligned}
$$

where $\Phi_{4}(t)=a_{11} \Phi_{2}(t)-a_{21} \Phi_{1}(t)$. Obviously, $\lim _{t \longrightarrow \infty}$ $\Phi_{4}(t)=0$.

From Lemma 4 , for any $\varepsilon>0$, there is a $T_{1}>0$ such that

$$
\begin{array}{r}
a_{11} \ln y_{2}(t) \leq\left(\Delta_{2}+a_{21} \varepsilon\right) t-\Delta \int_{0}^{t} y_{2}\left(s^{-}\right) \mathrm{d} s+\Phi_{4}(t), \\
\text { for } t>T_{1} .
\end{array}
$$

It follows from Lemma 3 that

$$
\lim _{t \rightarrow \infty} \bar{y}_{2}^{*}(t) \leq \frac{\Delta_{2}}{\Delta} \text { a.s. }
$$

Combining (43) and (46) leads to

$$
\lim _{t \rightarrow \infty} \overline{y_{2}(t)}=\frac{\Delta_{2}}{\Delta} \text { a.s. }
$$

Substituting (47) into (30) and using Lemma 2, we get

$$
\lim _{t \rightarrow \infty} \overline{y_{1}(t)}=\frac{\Delta_{1}}{\Delta} \text { a.s. }
$$

The proof is completed.

Next, let us consider model $(M)$.

Theorem 2. If $\Delta_{31}>0, \Delta_{41}>0$ hold, then for any initial $y(t)=\left\{\left(\xi_{1}(t), \xi_{2}(t)\right):-\tau \leq t \leq 0\right\} \in C\left([-\tau, 0] ; R_{+}^{2}\right)$, the solution $\left(y_{1}(t), y_{2}(t)\right)$ of model $(M)$ has the properties that

$$
\begin{aligned}
& \frac{\Delta_{31}}{\Delta} \leq{\overline{y_{1}(t)}}_{*} \leq{\overline{y_{1}(t)}}^{*} \leq \frac{\Delta_{32}}{\Delta}, \\
& \frac{\Delta_{41}}{\Delta} \leq{\overline{y_{2}(t)}}_{*} \leq{\overline{y_{2}(t)}}^{*} \leq \frac{\Delta_{42}}{\Delta} .
\end{aligned}
$$

That is to say, model $(M)$ will be persistence in mean.

Proof. Using Ito's formula to compute $\ln y_{i}(t)(i=1,2)$, we have

$$
t^{-1} \ln y_{1}(t)=b_{1}-r_{11} \overline{C_{1}(t)}-a_{11} \overline{y_{1}(t)}-a_{12} \overline{y_{2}(t)}+t^{-1} \Phi_{1}(t),
$$

$$
t^{-1} \ln y_{2}(t)=b_{2}-r_{21} \overline{C_{2}(t)}-a_{21} \overline{y_{1}(t)}-a_{22} \overline{y_{2}(t)}+t^{-1} \Phi_{2}(t) .
$$

Then,

$$
\begin{aligned}
& a_{11} t^{-1} \ln y_{2}(t)-a_{21} t^{-1} \ln y_{1}(t) \\
& \quad=a_{11}\left(b_{2}-r_{21} \overline{C_{2}(t)}\right)-a_{21}\left(b_{1}-r_{11} \overline{C_{1}(t)}\right)-\left(a_{11} a_{22}-a_{12} a_{21}\right) \overline{y_{2}(t)}+t^{-1} \Phi_{4}(t) \\
& \quad \geq a_{11}\left(b_{2}-r_{21}{\overline{C_{2}(t)}}^{*}\right)-a_{21}\left(b_{1}-r_{11} \overline{C_{1}(t)}\right)-\left(a_{11} a_{22}-a_{12} a_{21}\right) \overline{y_{2}(t)}+t^{-1} \Phi_{4}(t) \\
& \quad=\Delta_{41}-\Delta \overline{y_{2}(t)}+t^{-1} \Phi_{4}(t) .
\end{aligned}
$$

We can get from Lemma 3 that

$$
{\overline{y_{2}(t)}}_{*} \geq \frac{\Delta_{41}}{\Delta} \text { a.s. }
$$

For any $\varepsilon>0$, there is sufficiently $T_{2}>0$ such that

$$
a_{12} \overline{y_{2}(t)} \geq a_{12}{\overline{y_{2}(t)}}_{*}-\varepsilon \geq a_{12} \frac{\Delta_{41}}{\Delta}-\varepsilon, \quad t \geq T_{2} .
$$

Substituting (54) into (50), we get

$$
\begin{aligned}
t^{-1} \ln y_{1}(t) \leq & b_{1}-r_{11} \overline{C_{1}(t)} *-a_{12} \frac{\Delta_{41}}{\Delta}+\varepsilon \\
& -a_{11} \overline{y_{1}(t)}+t^{-1} \Phi_{1}(t) \\
= & \frac{a_{11} \Delta_{32}}{\Delta}+\varepsilon-a_{11} \overline{y_{1}(t)}+t^{-1} \Phi_{1}(t) .
\end{aligned}
$$


Therefore,

$$
{\overline{y_{1}(t)}}^{*} \leq \frac{\Delta_{32}}{\Delta} \text { a.s. }
$$

$$
\begin{aligned}
& a_{22} t^{-1} \ln y_{1}(t)-a_{12} t^{-1} \ln y_{2}(t) \\
& \quad=a_{22}\left(b_{1}-r_{11} \overline{C_{1}(t)}\right)-a_{12}\left(b_{2}-r_{21} \overline{C_{2}(t)}\right)-\left(a_{11} a_{22}-a_{12} a_{21}\right) \overline{y_{1}(t)}+t^{-1} \Phi_{3}(t) \\
& \quad \geq \Delta_{31}-\Delta \overline{y_{1}(t)}+t^{-1} \Phi_{3}(t) .
\end{aligned}
$$

In view of Lemmas 3 and 4 , we have

$$
{\overline{y_{1}(t)}}_{*} \geq \frac{\Delta_{31}}{\Delta} \text { a.s. }
$$

For any $\varepsilon>0$, there is a $T_{3}>0$ such that

$$
a_{21}{\overline{y_{1}(t)}} \geq a_{21}{\overline{y_{1}(t)}}_{*}-\varepsilon \geq a_{21} \frac{\Delta_{31}}{\Delta}-\varepsilon, \quad t \geq T_{3} .
$$

Substituting (59) into (51), we get

$$
\begin{aligned}
t^{-1} \ln y_{2}(t) \leq & b_{2}-r_{21} \overline{C_{2}(t)}{ }_{*}-a_{21} \frac{\Delta_{31}}{\Delta} \\
& +\varepsilon-a_{22} \overline{y_{2}(t)}+t^{-1} \Phi_{2}(t) \\
= & \frac{a_{22} \Delta_{42}}{\Delta}+\varepsilon-a_{22} \overline{y_{2}(t)}+t^{-1} \Phi_{2}(t),
\end{aligned}
$$

and then we have

$$
{\overline{y_{2}(t)}}^{*} \leq \frac{\Delta_{42}}{\Delta} .
$$

Combing (53), (56), (58), and (61) leads to the result. The proof is completed.

Remark 1. If the limit of $\overline{C_{1}(t)}$ and $\overline{C_{2}(t)}$ exist, that is, ${\overline{C_{1}}(t)}^{*}={\overline{C_{1}(t)}}_{*}$ and ${\overline{C_{2}}(t)}^{*}=\bar{C}_{2}(t)_{*}$, then Theorem 2 will be simplified as the following case.

If $\Delta_{31}>0, \Delta_{41}>0$ hold, then model $(M)$ will have the properties that

$$
\begin{aligned}
& \lim _{t \rightarrow \infty} \overline{y_{1}(t)}=\frac{\Delta_{31}}{\Delta}, \\
& \lim _{t \rightarrow \infty} \overline{y_{2}(t)}=\frac{\Delta_{41}}{\Delta} .
\end{aligned}
$$

\subsection{Nonpersistence}

Theorem 3. If $0<\left(a_{11} / a_{21}\right)\left(b_{2}-r_{21}{\overline{C_{2}(t)}}_{*}\right)<b_{1}-r_{11}{\overline{C_{1}(t)}}^{*}$ $\leq b_{1}-r_{11} \bar{C}_{1}(t) *$ holds, then for any initial data $y(t)=\left\{\left(\xi_{1}(t), \xi_{2}(t)\right):-\tau \leq t \leq 0\right\} \in C\left([-\tau, 0]: R_{+}^{2}\right)$, the solution $\left(y_{1}(t), y_{2}(t)\right)$ of model $(M)$ has the properties that
Similar computation leads to

$$
\begin{aligned}
\frac{b_{1}-r_{11}{\overline{C_{1}(t)}}^{*}}{a_{11}} \leq{\overline{y_{1}(t)}}_{*} \leq{\overline{y_{1}(t)}}^{*} \leq \frac{b_{1}-r_{11}{\overline{C_{1}(t)}}_{*}}{a_{11}}, \\
\lim _{t \rightarrow \infty} y_{2}(t)=0 \text { a.s. }
\end{aligned}
$$

That is to say, model $(M)$ is nonpersistent.

Proof. From (50), we get

$t^{-1} \ln y_{1}(t) \leq b_{1}-r_{11} \overline{C_{1}(t)}{ }_{*}-a_{11} \overline{y_{1}(t)}+t^{-1} \Phi_{1}(t)$.

According to Lemma 3, we get

$$
{\overline{y_{1}(t)}}^{*} \leq \frac{b_{1}-r_{11}{\overline{C_{1}(t)}}_{*}}{a_{11}} \text { a.s. }
$$

We can easily compute

$$
\begin{aligned}
a_{11} t^{-1} & \ln y_{2}(t)-a_{21} t^{-1} \ln y_{1}(t) \\
= & a_{11}\left(b_{2}-r_{21} \overline{C_{2}(t)}\right)-a_{21}\left(b_{1}-r_{11} \overline{C_{1}(t)}\right) \\
& -\Delta \overline{y_{2}(t)}+t^{-1} \Phi_{4}(t) .
\end{aligned}
$$

By Lemma 4, for enough large $t$ and any $\varepsilon>0$, there is

$$
\frac{\ln y_{i}(t)}{t}<\varepsilon, \quad i=1,2 \text {. }
$$

Then,

$$
\begin{aligned}
a_{11} t^{-1} \ln y_{2}(t) \leq & a_{11}\left(b_{2}-r_{21}{\overline{C_{2}(t)}}_{*}\right)-a_{21}\left(b_{1}-r_{11}{\overline{C_{1}(t)}}^{*}\right) \\
& -\Delta \overline{y_{2}(t)}+t^{-1} \Phi_{4}(t) .
\end{aligned}
$$

By the assumption

$$
\frac{a_{11}}{a_{21}}\left(b_{2}-r_{21}{\overline{C_{2}(t)}}_{*}\right)<b_{1}-r_{11}{\overline{C_{1}(t)}}^{*},
$$

and Lemma 3, we obtain from (68) that

$$
\lim _{t \rightarrow \infty} y_{2}(t)=0 \text { a.s. }
$$

Substituting (70) into (50), then

$$
\begin{aligned}
t^{-1} \ln y_{1}(t) & =b_{1}-r_{11} \overline{C_{1}(t)}-a_{11} \overline{y_{1}(t)}+t^{-1} \Phi_{1}(t) \\
& \geq b_{1}-r_{11}{\overline{C_{1}(t)}}^{*}-a_{11} \overline{y_{1}(t)}+t^{-1} \Phi_{1}(t)
\end{aligned}
$$


From Lemma 3, we get

$$
{\overline{y_{1}(t)}}_{*} \geq \frac{b_{1}-r_{11}{\overline{C_{1}(t)}}^{*}}{a_{11}},
$$

and thus,

$$
\frac{b_{1}-r_{11}{\overline{C_{1}(t)}}^{*}}{a_{11}} \leq{\overline{y_{1}(t)}}_{*} \leq{\overline{y_{1}(t)}}^{*} \leq \frac{b_{1}-r_{11}{\overline{C_{1}(t)}}_{*}}{a_{11}} .
$$

The proof is finished.

Theorem 4. If $\left(a_{11} / a_{21}\right)\left(b_{2}-r_{21}{\overline{C_{2}(t)}}_{*}\right)<b_{1}-r_{11}{\overline{C_{1}(t)}}^{*}$ $\leq b_{1}-r_{11}{\overline{C_{1}(t)}}_{*}<0$ holds, then for any initial data $y(t)=$ $\left\{\left(\xi_{1}(t), \xi_{2}(t)\right):-\tau \leq t \leq 0\right\} \in C\left([-\tau, 0]: R_{+}^{2}\right)$, the solution $\left(y_{1}(t), y_{2}(t)\right)$ of model $(M)$ has the properties that

$$
\begin{aligned}
& \lim _{t \longrightarrow \infty} y_{1}(t)=0, \\
& \lim _{t \longrightarrow \infty} y_{2}(t)=0 \text { a.s. }
\end{aligned}
$$

That is to say, model $(M)$ is nonpersistent.

Proof. Under the assumption that $b_{1}-r_{11}{\overline{C_{1}(t)}}_{*}<0$, we can get from Lemma 3 and (64) that

$$
\lim _{t \rightarrow \infty} y_{1}(t)=0 \text { a.s. }
$$

Substituting (75) into (51) reads

$$
\begin{aligned}
t^{-1} \ln y_{2}(t) & =b_{2}-r_{21} \overline{C_{2}(t)}-a_{22} \overline{y_{2}(t)}+t^{-1} \Phi_{2}(t) \\
& \leq b_{2}-r_{21}{\overline{C_{2}(t)}}_{*}-a_{22} \overline{y_{2}(t)}+t^{-1} \Phi_{2}(t) .
\end{aligned}
$$

The assumption $\left(a_{11} / a_{21}\right)\left(b_{2}-r_{21}{\overline{C_{2}(t)}}_{*}\right)<b_{1}-$ $r_{11}{\overline{C_{1}(t)}}^{*}<0$ implies that $b_{2}-r_{21}{\overline{C_{2}(t)}}_{*}<0$. Then, from (76) and Lemma 3 , it is easy to verify that

$$
\lim _{t \longrightarrow \infty} y_{2}(t)=0 \text { a.s. }
$$

This completes the proof.

\subsection{Stable in Distribution}

Theorem 5. If all the assumptions hold, then model (M) is stable in distribution.

Proof. The proof of this result is divided into three steps. Step 1 . We first prove model $(M)$ is globally attractive.

Let $\quad y(t)=\left(y_{1}(t ; \varphi), y_{2}(t ; \varphi)\right), y^{\wedge}(t)=\left(y_{1}\left(t ; \varphi^{\wedge}\right)\right.$, $\left.y_{2}\left(t ; \varphi^{\wedge}\right)\right)$ be any two solutions of $(M)$ with initial data $\varphi, \varphi^{\wedge} \in C\left([-\tau, 0], R_{+}^{2}\right)$, respectively. Denote $\tilde{y}_{i}(t)=y_{i}(t ; \varphi)$ $-y_{i}\left(t ; \varphi^{\wedge}\right), i=1,2$. We only need to verify that

$$
\begin{array}{r}
\lim _{t \longrightarrow+\infty} E\left|\tilde{y}_{i}(t)\right|=\lim _{t \longrightarrow+\infty} E\left|y_{i}(t ; \varphi)-y_{i}\left(t ; \varphi^{\wedge}\right)\right|=0, \\
i=1,2 .
\end{array}
$$

Define $V_{i}\left(t ; \varphi, \varphi^{\wedge}\right)=\left|\ln y_{i}(t ; \varphi)-\ln y_{i}\left(t ; \varphi^{\wedge}\right)\right|, i=1,2$. By Ito's formula, we get

$$
\begin{aligned}
L V_{1}\left(t ; \varphi, \varphi^{\wedge}\right) & =\operatorname{sign}\left(\widetilde{y}_{1}(t)\right)\left[-a_{11}\left(y_{1}(t ; \varphi)-y_{1}\left(t ; \varphi^{\wedge}\right)\right)-a_{12} \int_{-\tau_{2}}^{0}\left(y_{2}(t+\theta ; \varphi)-y_{2}\left(t+\theta ; \varphi^{\wedge}\right)\right) \mathrm{d} \mu_{2}(\theta)\right. \\
& \leq-a_{11}\left|\widetilde{y}_{1}(t)\right|+a_{12} \int_{-\tau_{2}}^{0}\left|\widetilde{y}_{2}(t+\theta)\right| \mathrm{d} \mu_{2}(\theta), \\
L V_{2}\left(t ; \varphi, \varphi^{\wedge}\right) & =\operatorname{sign}\left(\widetilde{y}_{2}(t)\right)\left[-a_{22}\left(y_{2}(t ; \varphi)-y_{2}\left(t ; \varphi^{\wedge}\right)\right)-a_{21} \int_{-\tau_{1}}^{0}\left(y_{1}(t+\theta ; \varphi)-y_{1}\left(t+\theta ; \varphi^{\wedge}\right)\right) \mathrm{d} \mu_{1}(\theta)\right. \\
& \leq-a_{22}\left|\widetilde{y}_{2}(t)\right|+a_{21} \int_{-\tau_{1}}^{0}\left|\tilde{y}_{1}(t+\theta)\right| \mathrm{d} \mu_{1}(\theta) .
\end{aligned}
$$

Let

$$
V\left(t ; \varphi, \varphi^{\wedge}\right)=\sum_{i=1}^{2} V_{i}\left(t ; \varphi, \varphi^{\wedge}\right)+V_{3}\left(t ; \varphi, \varphi^{\wedge}\right),
$$

where $\quad V_{3}\left(t ; \varphi, \varphi^{\wedge}\right)=a_{12} \int_{-\tau_{2}}^{0} \int_{t+\theta}^{t}\left|\widetilde{y}_{2}(s)\right| \mathrm{d} \mu_{2}(\theta) \mathrm{d} s+a_{21}$ $\int_{-\tau_{1}}^{0} \int_{t+\theta}^{t}\left|\tilde{y}_{1}(s)\right| \mathrm{d} \mu_{1}(\theta) \mathrm{d} s$.

Using Ito's formula to (80), we can easily compute that

$$
\begin{aligned}
L V\left(t ; \varphi, \varphi^{\wedge}\right) & =L V_{1}\left(t ; \varphi, \varphi^{\wedge}\right)+L V_{2}\left(t ; \varphi, \varphi^{\wedge}\right)+\frac{\mathrm{d} V_{3}\left(t ; \varphi, \varphi^{\wedge}\right)}{\mathrm{d} t} \\
& \leq-\left(a_{11}-a_{21} \int_{-\tau_{1}}^{0} \mathrm{~d} \mu_{1}(\theta)\right)\left|\tilde{y}_{1}(t)\right|-\left(a_{22}-a_{12} \int_{-\tau_{2}}^{0} \mathrm{~d} \mu_{2}(\theta)\right)\left|\tilde{y}_{2}(t)\right|
\end{aligned}
$$


According to (81), we get

$$
\begin{aligned}
E\left(V\left(t ; \varphi, \varphi^{\wedge}\right)\right) \leq & V\left(0 ; \varphi, \varphi^{\wedge}\right)-\left(a_{11}-a_{21} \int_{-\tau_{1}}^{0} \mathrm{~d} \mu_{1}(\theta)\right) \int_{0}^{t} E\left|\widetilde{y}_{1}(s)\right| \mathrm{d} s \\
& -\left(a_{22}-a_{12} \int_{-\tau_{2}}^{0} \mathrm{~d} \mu_{2}(\theta)\right) \int_{0}^{t} E\left|\widetilde{y}_{2}(s)\right| \mathrm{d} s
\end{aligned}
$$

which means

$$
\begin{aligned}
& E\left(V\left(t ; \varphi, \varphi^{\wedge}\right)\right)+\left(a_{11}-a_{21} \int_{-\tau_{1}}^{0} d \mu_{1}(\theta)\right) \int_{0}^{t} E\left|\widetilde{y}_{1}(s)\right| \mathrm{d} s+\left(a_{22}-a_{12} \int_{-\tau_{2}}^{0} \mathrm{~d} \mu_{2}(\theta)\right) \int_{0}^{t} E\left|\widetilde{y}_{2}(s)\right| \mathrm{d} s \\
& \quad \leq V\left(0 ; \varphi, \varphi^{\wedge}\right)<+\infty .
\end{aligned}
$$

Consequently,

$$
E\left(B_{i}(t)\right)=0, E\left(R_{i}(t)\right)=0, \quad i=1,2 .
$$

$$
E\left|\widetilde{y}_{i}(t)\right| \in L^{1}[0,+\infty), \quad i=1,2 .
$$

Therefore,

Furthermore, considering the continuity of $E\left(y_{i}(t)\right)$, $i=1,2$, and combining $(M)$, we have

$$
E\left(y_{1}(t)\right)=y_{1}(0)+\int_{0}^{t} E\left[r_{10} y_{1}(s)-r_{11} C_{1}(t) y_{1}(s)-a_{11} y_{1}^{2}(s)-a_{12} y_{1}(s) \int_{-\tau_{2}}^{0} y_{2}(s+\theta) \mathrm{d} \mu_{2}(\theta)\right] \mathrm{d} s
$$

It is not difficult to see that $E\left(y_{1}(t)\right)$ is differential. From Lemma 2, we get

$$
\begin{aligned}
\frac{\mathrm{d} E\left(y_{1}(t)\right)}{\mathrm{d} t} & =E\left[r_{10} y_{1}(t)-r_{11} C_{1}(t) y_{1}(t)-a_{11} y_{1}^{2}(t)-a_{12} y_{1}(t) \int_{-\tau_{2}}^{0} y_{2}(t+\theta) \mathrm{d} \mu_{2}(\theta)\right] \\
& \leq E\left(y_{1}(t)\right) r_{10} \leq r_{10} K_{1}
\end{aligned}
$$

where $K_{1}$ is a positive constant. Therefore, $E\left(y_{1}(t)\right)$ is uniformly continuous. Similarly, we can also obtain that $E\left(y_{2}(t)\right)$ is uniformly continuous. By virtue of (84) and Barbalat's conclusion in [26], we get

$$
\lim _{t \longrightarrow+\infty} E\left|\tilde{y}_{i}(t)\right|=0, \quad i=1,2 .
$$

Step 2. For any $\varphi \in C\left([-\tau, 0], R_{+}^{2}\right)$, it is denoted by $p(t, \varphi, \mathrm{d} z)$ the transition probability of the process $z(t)$, $P\left(t, \varphi, R_{+}^{2}\right)$ the probability of $\left(y_{1}(t ; \varphi), y_{2}(t ; \varphi)\right)^{T} \in R_{+}^{2}$, and
$\mathscr{P}\left([-\tau, 0], R_{+}^{2}\right)$ the space of all probability measures on $C\left([-\tau, 0], R_{+}^{2}\right)$. For any $P_{1}, P_{2} \in \mathscr{P}\left([-\tau, 0], R_{+}^{2}\right)$, define

$$
\mathrm{d}_{\mathrm{BL}}\left(P_{1}, P_{2}\right)=\sup _{g \in \mathrm{BL}}\left|\int_{R_{+}^{2}} g(z) P_{1}(\mathrm{~d} z)-\int_{R_{+}^{2}} g(z) P_{2}(\mathrm{~d} z)\right|
$$

where $\quad \mathrm{BL}=\left\{g: C\left([-\tau, 0], R_{+}^{2}\right) \longrightarrow R:\left|g\left(z_{1}\right)-g\left(z_{2}\right)\right| \leq\right.$ $\left.\left\|z_{1}-z_{2}\right\|,|g(\cdot)| \leq 1\right\}$. Thanks to Lemma 2 and Chebyshev's inequality, for any $\varphi \in C\left([-\tau, 0], R_{+}^{2}\right)$, the family $p(t, \varphi, \mathrm{d} z)$ 


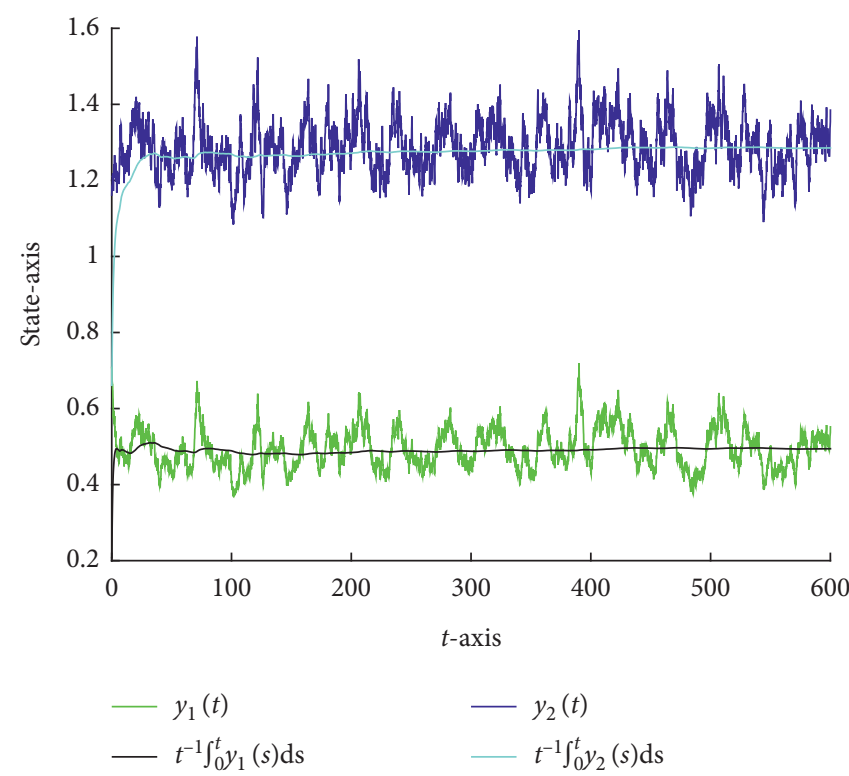

(a)

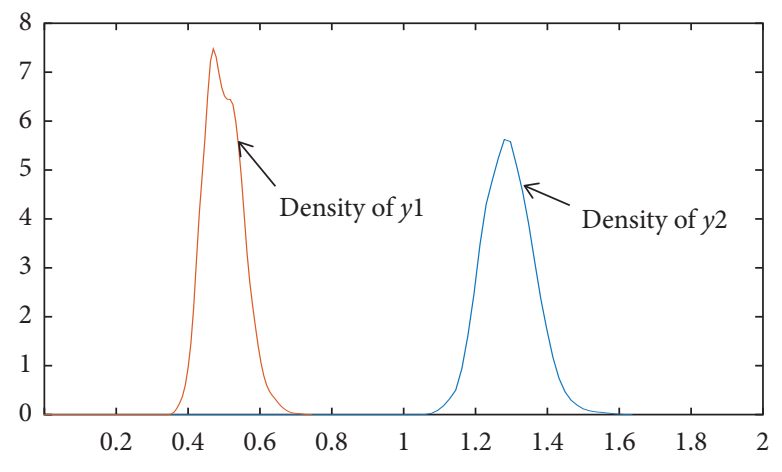

(b)

Figure 1: The persistent in mean of model $\left(M_{0}\right)$ with $\gamma_{1}(u)=1.0187, \gamma_{2}(u)=0.1332$. (a) The time series of $y_{1}, y_{2}$, respectively; (b) the probability density function of $\gamma_{1}(u)=1.0429, \gamma_{2}(u)=1.6876$, respectively.

is tight. That is, for any $\varepsilon \in(0,1)$, there exists a compact We compute subset $D \subseteq R_{+}^{2}$ such that $P(t, \varphi, D) \geq 1-\varepsilon$ for any $t \geq 0$..

$$
\begin{aligned}
\mathrm{d}_{\mathrm{BL}} & (p(t+s, \varphi, \cdot), p(t, \varphi, \cdot)) \\
= & \sup _{g \in \mathrm{BL}}\left|\int_{R_{+}^{2}} g(z(t+s ; \varphi)) p(t+s, \varphi, \mathrm{d} z)-\int_{R_{+}^{2}} g(z(t ; \varphi)) p(t, \varphi, \mathrm{d} z)\right| \\
= & \sup _{g \in \mathrm{BL}}|E[g(z(t+s ; \varphi))]-E[g(z(t ; \varphi))]| \\
= & \sup _{g \in \mathrm{BL}}\left|E\left[E\left[g(z(t+s ; \varphi)) \mid \mathscr{F}_{s}\right]\right]-E[g(z(t ; \varphi))]\right| \\
= & \sup _{g \in \mathrm{BL}}\left|\int_{R_{+}^{2}} E[g(z(t ; \psi))] p(s, \varphi, \mathrm{d} \psi)-E[g(z(t ; \varphi))]\right| \\
= & \sup _{g \in \mathrm{BL}}\left|\int_{R_{+}^{2}} E[g(z(t ; \psi))-g(z(t ; \varphi))] p(s, \varphi, \mathrm{d} \psi)\right| \\
\leq & \sup _{g \in \mathrm{BL}} \int_{R_{+}^{2}} E[|g(z(t ; \psi))-g(z(t ; \varphi))|] p(s, \varphi, \mathrm{d} \psi) \\
\leq & \sup _{g \in \mathrm{BL}} \int_{U_{B}} E[|g(z(t ; \psi))-g(z(t ; \varphi))|] p(s, \varphi, \mathrm{d} \psi) \\
& +\sup _{g \in \mathrm{BL}} \int_{R_{+}^{2} \backslash U_{B}} E[|g(z(t ; \psi))-g(z(t ; \varphi))|] p(s, \varphi, \mathrm{d} \psi),
\end{aligned}
$$

where $U_{B}=\left\{(x, y)^{T} \in R_{+}^{2}: \sqrt{x^{2}+y^{2}} \leq B\right\}$.

$$
\begin{gathered}
\sup _{g \in \mathrm{BL}} \int_{R_{+}^{2} \backslash U_{B}} E[|g(z(t ; \psi))-g(z(t ; \varphi))|] \\
\cdot p(s, \varphi, \mathrm{d} \psi) \leq 2 P\left(s, \varphi, R_{+}^{2} \backslash U_{B}\right) \leq 2 \varepsilon .
\end{gathered}
$$

Therefore, for sufficiently large $t$ and any $\varepsilon>0$, we can derive that

$$
\mathrm{d}_{\mathrm{BL}}(p(t+s, \varphi, \cdot), p(t, \varphi, \cdot)) \leq 3 \varepsilon
$$

That is to say, $\{p(t, \varphi, \cdot): t \geq 0\}$ is Cauchy in $\mathscr{P}\left([-\tau, 0], R_{+}^{2}\right)$ with initial data $\varphi \in C\left([-\tau, 0], R_{+}^{2}\right)$.

Step 3. We prove $\lim _{t \rightarrow+\infty} \mathrm{d}_{\mathrm{BL}}(p(t, \varphi, \cdot), v(\cdot))=0$.

According to (92), for $\varphi_{0} \in C\left([-\tau, 0], R_{+}^{2}\right)$, $\left\{p\left(t, \varphi_{0}, \cdot\right): t \geq 0\right\}$ is Cauchy in $\mathscr{P}\left([-\tau, 0], R_{+}^{2}\right)$, then there exists a unique $v(\cdot)$ such that 


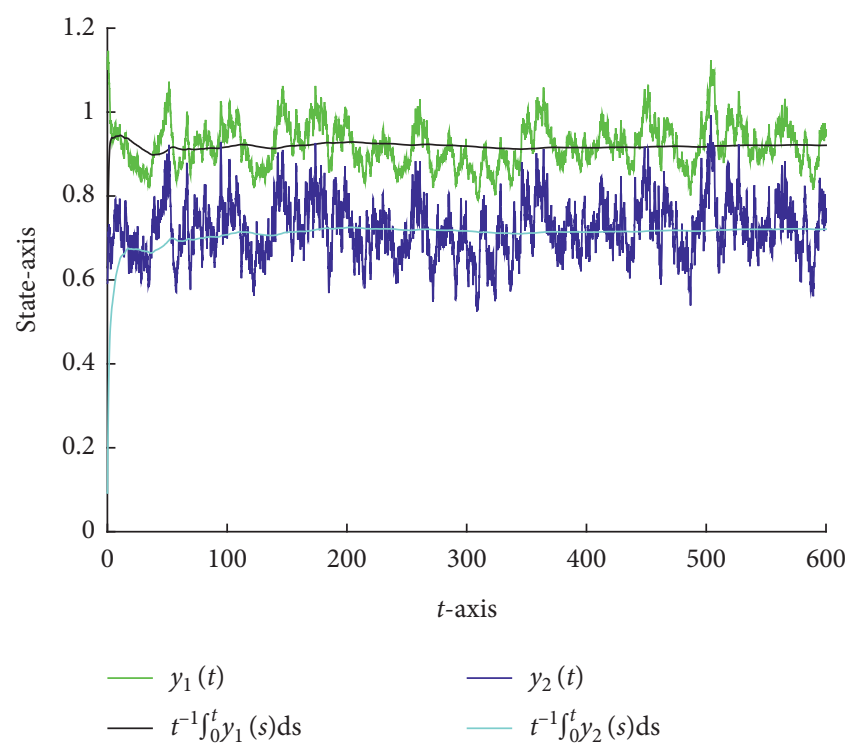

(a)

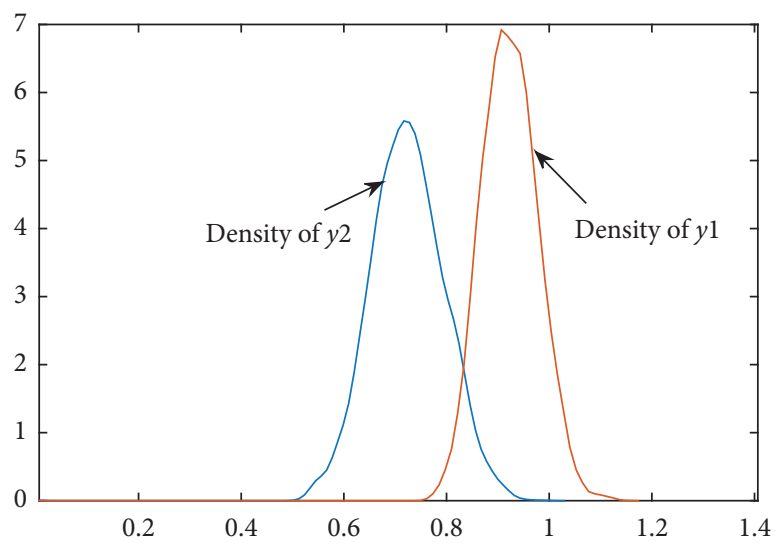

(b)

FIgURe 2: The persistent in mean of model $(M)$ with $\gamma_{1}(u)=0.1303, \gamma_{2}(u)=0.1837$. (a) Time series of $y_{1}$ and $y_{2}$; (b) the probability density function of $y_{1}$ and $y_{2}$.

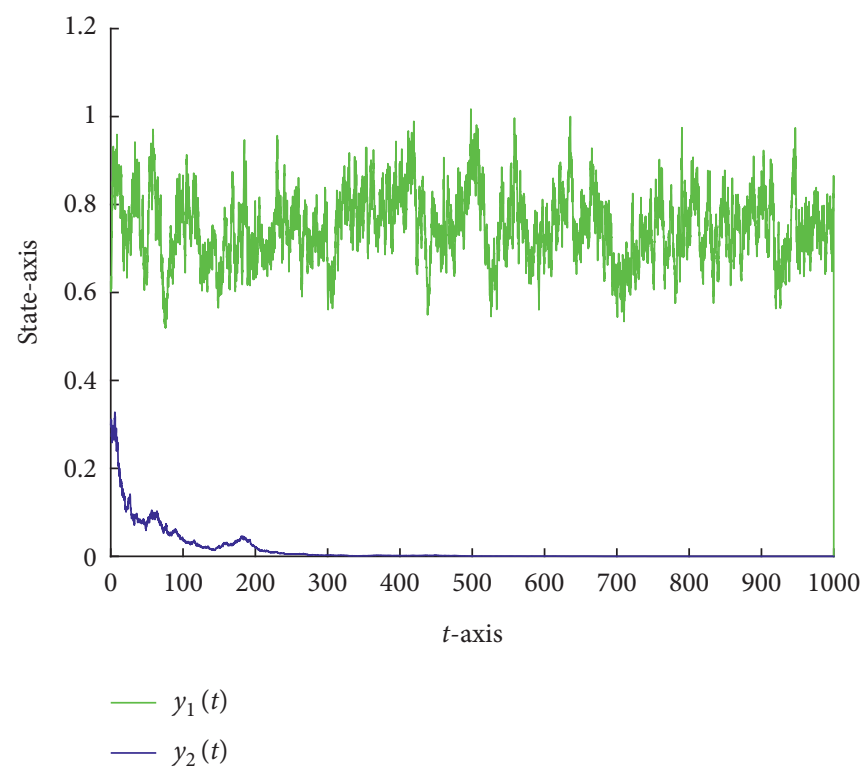

FIgURE 3: The nonpersistent in mean for model $(M)$ with $\gamma_{1}(u)=0.1303$,

$$
\lim _{t \rightarrow+\infty} \mathrm{d}_{\mathrm{BL}}\left(p\left(t, \varphi_{0}, \cdot\right), v(\cdot)\right)=0 .
$$

By virtue of (78), we derive $\gamma_{2}(u)=0.1837$. That is, specie $y_{2}$ will die out at some point.

$$
\begin{aligned}
\lim _{t \longrightarrow+\infty} \mathrm{d}_{\mathrm{BL}}\left(p(t, \varphi, \cdot), p\left(t, \varphi_{0}, \cdot\right)\right) & =\sup _{g \in B L}\left|E[g(z(t ; \varphi))]-E\left[g\left(z\left(t ; \varphi_{0}\right)\right)\right]\right| \\
& \leq \sup _{g \in B L} E\left[g(z(t ; \varphi))-g\left(z\left(t ; \varphi_{0}\right)\right)\right] \\
& \leq \lim _{t \rightarrow+\infty} E\left[\left\|z(t ; \varphi)-z\left(t ; \varphi_{0}\right)\right\|\right] \\
& =0 .
\end{aligned}
$$




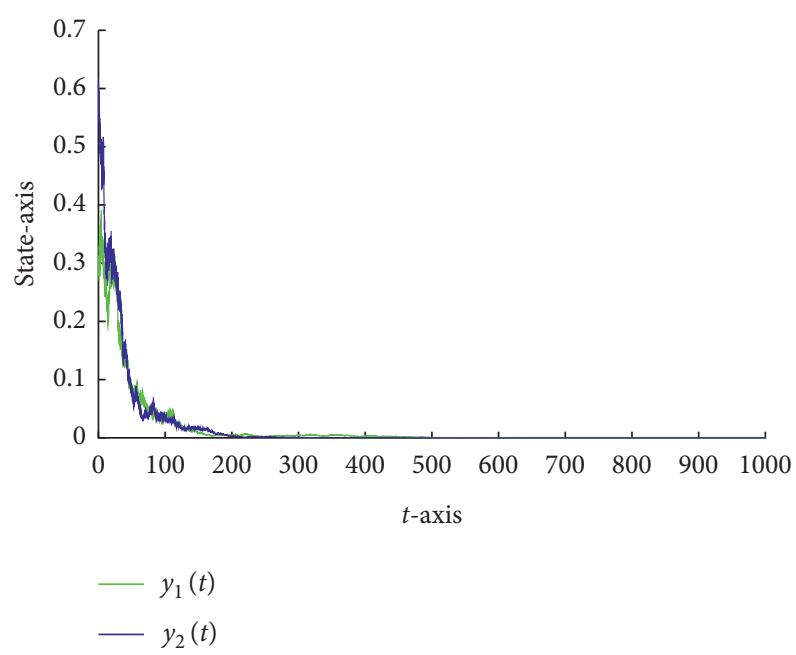

Figure 4: The extinction of model $(M)$ with $\gamma_{1}(u)=1.0429, \gamma_{2}(u)=1.6876$. Both species $y_{1}$ and $y_{2}$ will die out at some point.

By the triangle inequality and together with (93) and (94), we have

$$
\begin{aligned}
\lim _{t \longrightarrow+\infty} \mathrm{d}_{\mathrm{BL}}(p(t, \varphi, \cdot), v(\cdot)) \leq & \lim _{t \longrightarrow+\infty} \mathrm{d}_{\mathrm{BL}}\left(p(t, \varphi, \cdot), p\left(t, \varphi_{0}, \cdot\right)\right) \\
& +\lim _{t \longrightarrow+\infty} \mathrm{d}_{\mathrm{BL}}\left(p\left(t, \varphi_{0}, \cdot\right), v(\cdot)\right) \\
= & 0 .
\end{aligned}
$$

This completes the proof.

\section{Numerical Simulations}

In this section, some numerical simulations are given to verify our theoretical results. Take $a_{11}=0.52, a_{12}=0.02$, $a_{21}=0.03, a_{22}=0.8, r_{10}=0.71, r_{20}=1.35, \sigma_{1}^{2}=0.22, \sigma_{2}^{2}=$ $0.59, \tau_{1}=0.1, \tau_{2}=0.1$. It is easy to check that $\Delta=0.4154$, which means Assumption 2 holds.

(1) Set $\gamma_{1}(u)=1.0187, \gamma_{2}(u)=0.1332$, and then it is easy to count that $\Delta_{1}=0.2061, \Delta_{2}=0.5358$. Theorem 1 implies $\lim _{t \rightarrow \infty} \overline{y_{1}(t)}=0.4961, \lim _{t \rightarrow \infty}$ $\overline{y_{2}(t)}=1.2900$, that is, the species are both persistent in mean. Simulation also validates the result, see Figure 1.

(2) Set $\gamma_{1}(u)=0.1303, \gamma_{2}(u)=0.1837, r_{11}=0.48, r_{21}=$ $0.86, C_{1}(t)=0.1+0.1 \sin t, C_{2}(t)=0.4+0.1 \sin t$. By computation, we have $\Delta_{31}=0.3813, \Delta_{41}=0.2994$. By Theorem 2 and Remark 1, model $(M)$ is persistent in mean, shown in Figure 2.

(3) Set $\gamma_{1}(u)=0.5528, \gamma_{2}(u)=1.0391, r_{11}=0.48, r_{21}=$ $0.86, C_{1}(t)=0.1+0.1 \sin t, C_{2}(t)=0.4+0.1 \sin t$, and then these parameters satisfy the conditions of Theorem 3. By Theorem 3, we know that $y_{1}$ is permanent, but $y_{2}$ will die out, see Figure 3 .

(4) Set $\gamma_{1}(u)=1.0429, \gamma_{2}(u)=1.6876, r_{11}=0.1, r_{21}=$ $0.56, C_{1}(t)=0.4+0.1 \sin t, C_{2}(t)=0.4+0.1 \sin t$, $\sigma_{1}^{2}=0.87, \sigma_{2}^{2}=1.35$. Obviously, Theorem 4 shows that both $y_{1}$ and $y_{2}$ will die out, see Figure 4 .

\section{Conclusions}

The study of biological dynamics has been a popular topic in the field of biomathematics in recent years. With the development of economy, the environmental pollution is becoming more and more serious, which has become an important factor affecting the population relationship. Time delays are also important factors affecting the relationship. In this paper, we formulate a delayed predator-prey model with Lévy noise. Theorem 1 and 2 give the sufficient criteria of persistent in mean for cases $(M)$ and $\left(M_{0}\right)$, respectively. Theorem 3 and 4 obtain the sufficient conditions of nonpersistence. Theorem 5 investigates the stable in distribution. Finally, numerical simulations are given to validate our conclusion.

In view of the complexity of the environments, other factors such as the telephone noise and impulsive input may bring important influence to the dynamics, which needs further research in the future.

\section{Data Availability}

No data were used to support the findings of this study.

\section{Conflicts of Interest}

The authors declare that they have no conflicts of interest.

\section{Acknowledgments}

This work was supported by the National Natural Science Foundation of China (11861027).

\section{References}

[1] S. Ahmad and I. M. Stamova, "Almost necessary and sufficient conditions for survival of species," Nonlinear Analysis: Real World Applications, vol. 5, no. 1, pp. 219-229, 2004.

[2] R. M. May, Stability and Complexity in Model Ecosystem, Princeton University Press, Princeton, NJ, USA, 2001. 
[3] A. Szolnoki and M. perc, "Reward and cooperation in the spatial public goods game," Europhysics Letters, vol. 92, Article ID 38003, 2010.

[4] R. T. Paine, "Road maps of interactions or grist for theoretical development?" Ecology, vol. 69, no. 6, pp. 1648-1654, 1988.

[5] L. J. Zhang, "On a stochastic lotka-volterra competitive system with distributed delay and general Levy jumps," Mathematical Problems in Engineering, vol. 2016, Article ID 3407463, 9 pages, 2016.

[6] S. Wang, G. Hu, and L. Wang, "Stability in distribution of a stochastic competitive lotka-volterra system with S-type distributed time delays," Methodology and Computing in Applied Probability, vol. 20, no. 4, pp. 1241-1257, 2018.

[7] C. Ji, D. Jiang, and N. Shi, "Analysis of a predator-prey model with modified Leslie-Gower and Holling-type II schemes with stochastic perturbation," Journal of Mathematical Analysis and Applications, vol. 359, no. 2, pp. 482-498, 2009.

[8] C. Ji, D. Jiang, and X. Li, "Qualitative analysis of a stochastic ratio-dependent predator-prey system," Journal of Computational and Applied Mathematics, vol. 235, no. 5, pp. 13261341, 2011.

[9] C. Ji, D. Jiang, and N. Shi, "A note on a predator-prey model with modified Leslie-Gower and Holling-type II schemes with stochastic perturbation," Journal of Mathematical Analysis and Applications, vol. 377, no. 1, pp. 435-440, 2011.

[10] Y. Huang, Q. Liu, and Y. Liu, "Global asymptotic stability of a general stochastic Lotka-Volterra system with delays," Applied Mathematics Letters, vol. 26, no. 1, pp. 175-178, 2013.

[11] G. Q. Cai and Y. K. Lin, "Stochastic analysis of predator-prey type ecosystems," Ecological Complexity, vol. 4, no. 4, pp. 242-249, 2007.

[12] X. Mao, S. Sabanis, and E. Renshaw, "Asymptotic behaviour of the stochastic Lotka-Volterra model," Journal of Mathematical Analysis and Applications, vol. 287, no. 1, pp. 141-156, 2003.

[13] R. Manivannan, R. Samidurai, J. Cao, and M. Perc, “Design of resilient reliable dissipativity control for systems with actuator faults and probabilistic time-delay signals via sampled-data approach," IEEE Transactions on Systems, Man, and Cybernetics: Systems, vol. 50, no. 11, pp. 4243-4255, 2020.

[14] L. Wang, R. Zhang, and Y. Wang, "Global exponential stability of reaction-diffusion cellular neural networks with S-type distributed time delays," Nonlinear Analysis: Real World Applications, vol. 10, pp. 1101-1113, 2009.

[15] L. Wang and D. Xu, "Global asymptotic stability of bidirectional associative memory neural networks with S-type distributed delays," International Journal of Systems Science, vol. 33, no. 11, pp. 869-877, 2002.

[16] M. Liu and K. Wang, "Analysis of a stochastic autonomous mutualism model," Journal of Mathematical Analysis and Applications, vol. 402, no. 1, pp. 392-403, 2013.

[17] Q. Liu, Q. Chen, and Z. Liu, "Analysis on stochastic delay Lotka-Volterra systems driven by Lévy noise," Applied Mathematics and Computation, vol. 235, pp. 261-271, 2014.

[18] M. Liu and K. Wang, "Stochastic Lotka-Volterra systems with Lévy noise," Journal of Mathematical Analysis and Applications, vol. 410, no. 2, pp. 750-763, 2014.

[19] R. Wu, X. Zou, and K. Wang, "Asymptotic properties of stochastic hybrid Gilpin-Ayala system with jumps," Applied Mathematics and Computation, vol. 249, pp. 53-66, 2014.

[20] M. Liu and K. Wang, "Dynamics of a Leslie-Gower Hollingtype II predator-prey system with Lévy jumps," Nonlinear Analysis: Theory, Methods \& Applications, vol. 85, pp. 204$213,2013$.
[21] M. Liu and C. Bai, "Analysis of a stochastic tri-trophic foodchain model with harvesting," Journal of Mathematical Biology, vol. 73, no. 3, pp. 597-625, 2016.

[22] J. Wu, "Dynamics of a two-predator one-prey stochastic delay model with Lévy noise," Physica A: Statistical Mechanics and Its Applications, vol. 539, p. 122910, 2020.

[23] A. Muhammashaji and Z. Teng, "On a two species stochastic Lotka-Volterra competition system," Journal of Dynamical and Control Systems, vol. 21, pp. 495-511, 2015.

[24] N. Tuerxun, X. Abdurahman, and Z. Teng, "Global dynamics and optimal harvesting in a stochastic two-predators one-prey system with distributed delays and Lévy noise," Journal of Biological Dynamics, vol. 14, no. 1, pp. 32-56, 2020.

[25] Q. Han, D. Jiang, and C. Ji, "Analysis of a delayed stochastic predator-prey model in a polluted environment," Applied Mathematical Modelling, vol. 38, no. 13, pp. 3067-3080, 2014.

[26] I. Barbalat, "Systems d'equations differentielles d'oscillations nonlineaires," Revue Roumaine des Mathematiques Pures et Appliquees, vol. 4, pp. 267-270, 1959. 